\title{
数值天气预报涡度方程差分格式的 守恒性和稳定性
}

\author{
郭本 瑜 \\ (上海科学技术大学)
}

数值天气预报中差分方法的主要问题是:(1)构思格式的原则;(2)格式对初始条件误差和 计算误差的稳定性; (3)边界条件类型及其误差对局部地区预报值的影响; (4) Lorenz ${ }^{[1]}$ 所指出 的可预报期. 作者和 Morton 等都研究了格式的守恒性, 并把它作为加强稳定性的手段 ${ }^{[2-6]}$. 本文则以二维洞度方程为例,比较全面地讨论了上述诸问题.

\section{一、格式的守恒性}

$R$ 和 $\Gamma$ 表示 $\left(x_{1}, x_{2}\right)$ 平面中的有界区域及其边界, $\xi, \psi$ 是绝对洞度及流函数， $f$ 是柯氏参 数,那么最简单的正压模式是

$$
\begin{cases}\frac{\partial \xi}{\partial t}-\frac{\partial \psi}{\partial x_{2}} \frac{\partial \xi}{\partial x_{1}}+\frac{\partial \psi}{\partial x_{1}} \frac{\partial \xi}{\partial x_{2}}=0, & R \times(0, T], \\ \nabla^{2} \psi-\xi+f=0, & R \times(0, T] .\end{cases}
$$

记 $E_{R}(\xi, t)-\iint_{R} \xi^{i}\left(x_{1}, x_{2}, t\right) d x_{1} d x_{2}$, 则由(1)式得守恒律

$$
E_{i}(\xi, t)-E_{i}(\xi, 0)=\int_{0}^{t} \int_{\Gamma} \xi^{i}\left(\frac{\partial \psi}{\partial x_{1}} d x_{1}+\frac{\partial \psi}{\partial x_{2}} d x_{2}\right) d \tau, \quad i=1,2 .
$$

为了计算 (1)式, 用 $h$ 表示空间方向的网格步长, $Q$ 是网格点, $R_{h}$ 是网格区域的内点集合, $\Gamma_{h}$ 是其边界, $R_{h}^{*}$ 是与 $\Gamma_{b}$ 相距 $h$ 的内点集合. $\tau$ 表示时间方向的网格步长, $\lambda=\tau h^{-2}, W(k)$ 或 $W$ 都表示定义在 $R_{h}+\Gamma_{h}$ 上的网格函数在 $k \tau$ 时刻的值, $\bar{W}=\bar{W}(k)=W(k+1) . W_{x_{i}}$, $W_{\bar{x}_{j}}, W_{z}$ 分别表示 $x_{j}$ 方向的向前、向后和中心差商, $W_{n}$ 表示外法向差商. $\Delta_{x_{j}}^{v} W=\frac{1}{2}\left(\nu W_{x_{j}}\right)_{\bar{x}_{j}}$ $+\frac{1}{2}\left(\nu W_{\bar{x}_{j}}\right)_{x_{i}}, \Delta^{\nu} W=\sum_{j=1}^{2} \Delta_{x_{j}}^{v} W$. 当 $\nu \equiv 1$ 时, 用 $\Delta W$ 简记 $\Delta^{\nu} W$.

又定义 $(W, V)=\sum_{Q \in R_{h}} h^{2} W(Q) V(Q),\|W\|^{2}=(W, W)$,

$$
\|W\|_{1 v}^{2}=\frac{1}{2} \sum_{j=1}^{2}\left(\left\|\sqrt{\nu} W_{x_{j}}\right\|^{2}+\left\|\sqrt{\nu} W_{\bar{x}_{j}}\right\|^{2}\right), \quad\|W\|_{\Gamma_{h}}^{2}=\sum_{Q \in \Gamma_{h}} h W_{0}^{2}(Q) .
$$

由于 $h \neq 0$, 差分格式很难同时模拟 $E_{1}, E_{2}$ 的守恒性. Lax 型格式模拟了 $E_{1}$, 本文模拟 $E_{2}$, 为此定义下列差分算子:

本文 1977 年 8 月 11 日收到. 


$$
\begin{aligned}
& J_{x_{1 \cdot 1}}^{+}(V, W)=V_{x_{1}} W_{\hat{x}_{2}}, \quad J_{x_{1 \cdot 1}}^{-}(V, W)=V_{\bar{x}_{1}} W_{\hat{x}_{2}}, \\
& J_{x_{1 \cdot 2}}^{+}(V, W)=\left(V W_{\hat{x}_{2}}\right)_{x_{1}}, \quad J_{x_{1 \cdot 2}}^{-}(V, W)=\left(V W_{\hat{x}_{2}}\right)_{\tilde{x}_{1}}, \\
& J_{x_{1,3}}^{+}(V, W)=-\left(V_{\hat{x}_{1}}, W\right)_{x_{1}}, J_{x_{1 \cdot 3}}^{-}(V, W)=-\left(V_{\hat{x}}, W\right)_{\tilde{x}_{1}}, \\
& J_{x_{2 \cdot 1}}^{+}(V, W)=-V_{x}, W_{\dot{x}_{1}}, J_{x_{2 \cdot 1}}^{-}(V, W)=-V_{\bar{x}_{2}} W_{k}, \\
& J_{x_{2 \cdot 2}}^{+}(V, W)=-\left(V W_{\hat{x}_{1}}\right)_{x_{2}}, \quad J_{x_{2 \cdot 2}}^{-}(V, W)=-\left(V W_{\hat{z}_{1}}\right)_{\bar{x}_{2}}, \\
& J_{x_{2 \cdot 3}}^{+}(V, W)=\left(V_{\hat{x}, W} W\right)_{x,}, \quad J_{x_{2 \cdot 3}}^{-}(V, W)=\left(V_{\hat{x}_{1}} W\right)_{\bar{x}_{2}} \text {, } \\
& J_{x_{i} \cdot i}(V, W)=\frac{1}{2} J_{x_{j, i}}^{+}(V, W)+\frac{1}{2} J_{x_{j \cdot i}}^{-}(V, W) \text {, } \\
& J_{i}^{+}(V, W)=\sum_{j=1}^{2} J_{x_{j \cdot i}}^{+}(V, W), \quad J_{i}^{-}(V, W)=\sum_{j=1}^{2} J_{x_{j \cdot i}}^{-}(V, W), \\
& J^{+}(V, W)=\sum_{i=1}^{3} \alpha_{i} J_{i}^{+}(V, W), J^{-}(V, W)=\sum_{i=1}^{3} \alpha_{i} J_{i}^{-}(V, W),
\end{aligned}
$$

其中 $\quad \alpha_{i} \geqslant 0, \sum_{i=1}^{3} \alpha_{i}=1$,

$$
J_{x_{j}}(V, W)=\sum_{i=1}^{3} \alpha_{i} J_{x_{j} \cdot i}(V, W), J(V, W)=\sum_{j=1}^{2} J_{x_{j}}(V, W) .
$$

用 $\eta, p$ 和 $J(\eta, \varphi)$ 来逼近 $\xi, \psi$ 和(1)式中的 Jacobin 算子. 若 $\alpha_{1}=\alpha_{2}$, 则 $(\eta, J(\eta, \varphi))$ $=B(\eta, \varphi)$, 其中 $B(\eta, \varphi)$ 是(2)式右端的合理模拟. 在下列两种边界条件下, $B(\eta, \varphi)=0$.

1) $\eta, \varphi$ 在 $x_{i}$ 方向具有周期 $L_{i}, R_{h}$ 是长方形 $\left[0, L_{1}-h ; 0, L_{2}-h\right]$, 并在某内点处, $\varphi=$ 常数. 全球预报问题属于这一类.

2) 在边界上 $\eta$ 的流量为零.

设参数 $0 \leqslant \delta \leqslant 1$,计算( $(1)$ 式的基本格式是

$$
\left\{\begin{array}{l}
L_{1}(\eta, \varphi)=\eta_{t}-J\left(\eta+\delta \tau \eta_{t}, \varphi\right)=0, \\
L_{2}(\eta, \varphi)=\Delta \varphi-\eta+f=0 .
\end{array}\right.
$$

假定满足条件 1)或 2), 则当 $\alpha_{1}=\alpha_{2}, \delta=\frac{1}{2}$ 时, $\|\eta\|^{2}$ 严格守恒. 对于显式格式, $\delta=0$, 则 $\|\eta\|_{t}^{2}=\tau\left\|\eta_{t}\right\|^{2}$. 此即每计算一步都有虚假能量的微小增长,若积累值超过一定值,就会引起“能 量爆发”. 为了克服这一现象,可考虑有粘性的正压模式, 从而得到

$$
\left\{\begin{array}{l}
\frac{\partial \xi}{\partial t}-\frac{\partial \psi}{\partial x_{2}} \frac{\partial \xi}{\partial x_{1}}+\frac{\partial \psi}{\partial x_{1}} \frac{\partial \xi}{\partial x_{2}}-\frac{\partial}{\partial x_{1}}\left(\nu \frac{\partial \xi}{\partial x_{1}}\right)-\frac{\partial}{\partial x_{2}}\left(\nu \frac{\partial \xi}{\partial x_{2}}\right)=0, \\
\nabla^{2} \psi-\xi+f=0,
\end{array}\right.
$$

其中, $0 \leqslant \nu_{0} \leqslant v\left(x_{1}, x_{2}, t\right) \leqslant \nu_{1}$. 相应的差分格式是

$$
\left\{\begin{array}{l}
L_{3}(\eta, \varphi)=L_{1}(\eta, \varphi)-\Delta^{\nu}\left(\eta+\sigma \tau \eta_{t}\right)=0, \\
L_{2}(\eta, \varphi)=0
\end{array}\right.
$$

其中 $0 \leqslant \sigma_{0} \leqslant \sigma \leqslant \sigma_{1}$.

\section{二、格式的广义稳定性}

设有非定常差分格式, $L_{h r}(\eta(k))=f(k)$, 其中 $f$ 是定解条件, $\tilde{\eta}, \tilde{f}$ 表示 $\eta$ 和 $f$ 的计算 误差. 若对某种模 $\|\cdot\|_{n}$ 和 $\|\cdot\|_{t}$, 适当小的 $h, \tau$, 存在与 $h, \tau$ 无关的正数 $M, N$ 和常数 $S^{\prime}$, 便 


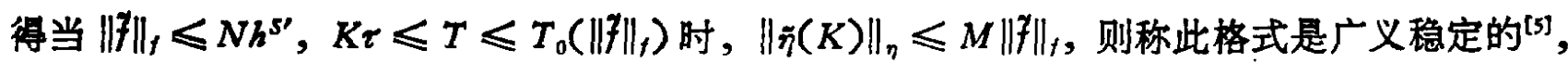
并称此类 $S^{\prime}$ 的下确界 $S$ 为广义稳定性指标, 这是一种 fuzzing 数学的概念.

若把 7 看作初始误差和计算误差,则 $S$ 越小计算就越稳定. 当 $S \leqslant 0$ 时, 只要 $\|7\|_{f} \leqslant N$, 计算就是稳定的. 若把 7 看作格式的形式適近误差, 且当 $h \rightarrow 0$ 时, $\| f_{f}=O\left(h^{s_{a}}\right)$, 则当 $S_{a}>0, s \leqslant s_{a}$ 时,格式就收玫. 近年来, Weinberger ${ }^{[7]}$ 等提出了差分格式优化问题,都致力于 增大线性格式的 $S_{a}$ 值. 作者认为非线性格式的 $S_{a}$ 一般较小,优化的主要目的在于下降 $S$ 值. 这样既加强稳定性，也保证在 $S_{a}$ 较小时,格式也是收玫的.

下面给出周期解问题 $S$ 的上界. 用 $\tilde{\eta}, \tilde{\varphi}, f_{i}$ 分别表示 $\eta, \varphi$ 和 (5) 式中第 $i$ 式的计算误 差. $a, b$ 是任意正数, $L, M, N$ 是与 $h, \tau$ 无关的正数, $m^{*}=\max \left\{2 \sigma(2 \sigma-1)^{-1}, a+1\right\}$, $m^{* *}(b)=\max \left\{\left[4+(32+2 b) \lambda v_{1} \sigma+4 a\right]\left[4+(32+2 b) \lambda_{\nu_{1}} \sigma-(16+b) \lambda v_{1}\right]^{-1}, a+1\right\}$. 条件 $(\sigma, \lambda, b)$ 表示或者 $\sigma_{0} \geqslant \frac{1}{2}$, 或者 $\lambda<4 \nu_{1}^{-1}(16+b-32 \sigma-2 b \sigma)^{-1}$. 条件 $(\sigma, \lambda, \delta, b)$ 表示不仅上述条件满足, 而且当 $\sigma_{0}>\frac{1}{2}$ 时, $2 \delta>m^{*}$, 否则 $2 \delta>m^{* *}(b)$. 又记

$$
\|\tilde{\eta}(K)\|_{0 \oplus v}^{2}=\|\tilde{\eta}(K)\|^{2}+\tau \sum_{j=0}^{K-1}\|\tilde{\eta}(j)\|_{1 v}^{2},
$$

$$
\rho\left(\tilde{\eta}, \tilde{f}_{1}, \mathfrak{f}_{2}, \tilde{g}, K\right)=\|\tilde{\eta}(0)\|^{2}+\tau \sum_{j=0}^{K-1}\left(\left\|f_{1}(j)\right\|^{2}+\left\|\mathfrak{f}_{2}(j)\right\|^{2}+\|\tilde{g}(j)\|_{\Gamma_{h}}^{2}\right) .
$$

定五 1 若用(5)式计算周期解,条件 $(\sigma, \lambda, 0)$ 满足,那末

(1) 当 $\left\|f_{2}\right\|^{2} \leqslant N h^{2 s}, \rho=\rho\left(\tilde{\eta}, \mathfrak{f}_{1}, \mathfrak{f}_{2}, 0, K\right) \leqslant N h^{2 s}$ 时,对一切 $K \tau \leqslant T \leqslant T_{0}(\rho)$ 者, $\|\tilde{\gamma}(K)\|_{\omega_{\oplus \nu}}^{2} \leqslant M e^{L T} \rho$. 其中当 $\nu_{0}>0$ 或 $\alpha_{1}=\alpha_{2}$ 时, $s \leqslant 1$. 当 $\nu_{0}>0$, 且 $\alpha_{1}=\alpha_{2}$ 时, $s \leqslant 0$.

(2) 又若当 $h \rightarrow 0$ 时, $\left\|f_{2}\right\|^{2}=o\left(h^{2 s}\right), \rho\left(\tilde{\eta}, f_{1}, f_{2}, 0, K\right)=o\left(h^{2 s}\right)$, 则 $T_{0}$ 任意.

(3) 如果条件 $(\sigma, \lambda, \delta, 0)$ 也满足, 则对一切 $f_{2}, \rho$ 和 $T_{0},\|\tilde{\eta}(K)\|_{0 \oplus p}^{2} \leqslant M e^{L T} \rho$.

根据定理 1, 参数 $\alpha_{1}=\alpha_{2}$, 不仅合理地模拟了守恒律, 而且也使格式稳定. 此时即使不固 有平滑过程 $(\nu \equiv 0)$, 显式格式仍有 $s \leqslant 1$, 若 $\xi, \psi$ 适当光滑, 则格式(5)收玫. 若同时伴有全 场平滑 $\left(\nu_{0}>0\right)$, 则显式格式的 $s \leqslant 0$ ，因而不仅计算稳定，而且即使 $\xi ， \psi$ 发生某种弱间断， 格式(5)同样是收敛的. $\alpha_{1}=\alpha_{2}$ 的隐式格式具有通常的绝对稳定性.

\section{三、两步格式的守恒性与㻊定性}

也可用两步法来加强计算(1)式的稳定性,并提高波前分讋度.

方法 I

$$
\left\{\begin{array}{l}
\text { 预估值 } \eta^{*}=\eta+\tau J^{+}(\eta, \varphi), \\
\text { 校正值 } \bar{\eta}=\frac{1}{2}\left(\eta+\eta^{*}\right)+\frac{\tau}{2} J^{-}\left(\eta^{*}, \varphi\right), \alpha_{1}=\alpha_{2 。} .
\end{array}\right.
$$

它的解满足能量关系式 $\left\|\eta_{t}\right\|^{2}=\tau\left\|\eta_{t}\right\|^{2}-\tau\left\|J^{+}(\eta, \varphi)\right\|^{2}$. 由于 $\left\|\eta_{t}\right\|^{2}$ 大致与 $\left\|J^{+}(\eta, \varphi)\right\|^{2}$ 相当, 从而有效地抑制了虚假的能量增长. 可交换使用 $J^{+}$和 $J$ 的次序, 或者加权平均地使用.

方法 II $\left\{\begin{array}{l}\eta^{*}=\eta+\beta \tau J(\eta, \varphi)+\tau^{2} \gamma \Delta \eta, \gamma, \beta \geqslant 0, \\ \eta=\eta+\tau J\left(\eta^{*}, \varphi\right) .\end{array}\right.$

若 $\beta=\frac{1}{2}, \alpha_{2}=1, \gamma=\frac{h^{2}}{2 \tau^{2}}$, 此即 Lax-Wendroff 两步格式. 若 $\beta=1, \alpha_{2}=1, \gamma=0$, 此即 Matsnuo 格式. 
定理 2 若在(6)式中 $\gamma=0, \alpha_{1}=\alpha_{2}=\frac{1}{2}, \beta>\frac{3 a+1}{2}, a>0, \lambda<(2 \beta-3 a-1)^{\frac{1}{2}}$. $a^{\frac{1}{2}} \cdot\left(4 \beta(6 a+2)\|\varphi\|_{1}\right)^{-1}$, 则当 $\left\|\tilde{f}_{2}\right\|^{2} \leqslant N,\left\|\rho\left(\tilde{\eta}, \tilde{f}_{1}, \tilde{f}_{2}, 0, K\right)\right\|^{2} \leqslant N$ 时, 对 $K \tau \leqslant T \leqslant T_{0}(\rho)$ 者, $\|\tilde{\eta}(K)\|^{2} \leqslant M e^{L T} \rho$.

定理 2 中的格式，由于结合使用了加权平均守恒法及 Matsnuo 型格式，所以使无粘流显 式格式的 $s \leqslant 0$. 计算结果表明它优于 Lax-Wendroff 格式和通常的 Matsnuo 格式.

方法 III 把本文方法与 Splitting 方法相结合, 例如

$$
\left\{\begin{array}{l}
\eta^{*}=\eta+\tau J_{x_{1}}\left(\frac{\eta^{*}+\eta}{2}, \varphi\right), \\
\ddot{\eta}=\eta^{*}+\tau J_{x_{2}}\left(\frac{\bar{\eta}+\eta^{*}}{2}, \varphi\right), \quad \text { 其中 } \alpha_{1}=\alpha_{2} .
\end{array}\right.
$$

显然 $\|\tilde{\eta}\|^{2}=\|\eta\|^{2}$ ，因此能量严格守恒，且又可用追赶法显式地求解. Nitta ${ }^{[8]}$ 和Temperton ${ }^{[9]}$ 格式也属于此类多步格式.

\section{四、边值条件及其误茎的影响}

假设局部地区预报的边界条件是 $\eta=g_{1}, \varphi=g_{0}, g_{i}$ 的计算误差是 $\tilde{g}_{i}$, 且不妨假设 $\tilde{\mathrm{g}}_{0}=0$. 又记 $\nu^{*}=\min _{Q \in R_{h}^{*}+\Gamma_{h}} \nu(Q), a^{*}=\max _{Q \in R_{h}^{*}} \frac{\nu(Q)}{\nu\left(Q^{\prime}\right)}$, 其中 $Q^{\prime}$ 是 $\Gamma_{h}$ 上距 $Q$ 为 $h$ 的点.

定理 3 假设采用(5)式计算上述初值一边值问题，条件 $(\sigma, \lambda, 1)$ 满足，且当 $\tilde{\mathbf{g}}_{1} 0$ 时, $a^{*}$ 有界, $v^{*}>0$, 那末

（1）当 $\left\|\tilde{f}_{2}\right\|^{2} \leqslant N h^{2 s} ，\left\|\tilde{g}_{1}\right\|^{2} \leqslant N h^{2 s+1}, \rho^{*}=\rho\left(\tilde{\eta}, \tilde{f}_{1}, \tilde{f}_{2}, h^{-\frac{1}{2}} \tilde{g}_{1}, K\right) \leqslant N h^{2 s}$ 时, 对一切 $K \tau \leqslant T \leqslant T_{0}\left(\rho^{*}\right)$ 者, $\|\tilde{\eta}(K)\|_{0 \oplus \nu}^{2} \leqslant M e^{L T} \rho^{*}$, 其中 $S$ 值同定理 1 .

（2）若又满足条件 $(\sigma, \lambda, \delta, 1)$, 则当 $\left\|\tilde{g}_{1}\right\|^{2} \leqslant N h$ 时，上式就成立.

由此可知,(1)对于局部地区预报,参数 $\alpha_{1}=\alpha_{2}$, 仍然是优化的. (2)边值误差会显著影响 $s$ 值. 事实上, 若 $\tilde{g}_{1} \equiv 0$, 则当 $\left\|\tilde{f}_{2}\right\|^{2} \leqslant N h^{2 s},\|\tilde{\eta}(0)\|^{2} \leqslant N h^{2 s}$ 时, 计算就稳定. 否则尚要求 $\left\|\tilde{g}_{1}\right\|_{r_{h}}^{2} \leqslant N h^{2 s+1}$. (3)若 $\tilde{g}_{1} \equiv 0$, 即使不固有平滑过程(即 $\nu \equiv 0$ ), 计算仍收玫. 否则宜在边 界附近固有平滑过程. (4)周期解问题的 $S$ 值与 $\tilde{g}_{1} \equiv 0$ 时相当, 所以计算稳定,此与 Shapiro 经验一致 ${ }^{[2]}$. 此外, 当 $R_{h}$ 是长方形, $\tilde{g}_{1} \equiv 0$ 时,尚可证明,当 $\alpha_{1}=\alpha_{2}, v_{0}>0$ 时, $S \leqslant-0.5$, 当 $\alpha_{1} \neq \alpha_{2}, \nu_{0}>0$ 时, $S \leqslant 0$, 当 $\alpha_{1}=\alpha_{2}, \nu_{0}=0$ 时, $S \leqslant 0.5$. 因此, 合适的边界形状可 增强计算的稳定性. 又如果采用文献 [5]中的修正逆风法,其结果与定理 3 中的 $\alpha_{1} \neq \alpha_{2}$ 情况 相同. 建议在内点采用(5)式计算,在边界附近采用修正逆风法计算,在文献 $[10,11]$ 中也有类 似方法.

再考虑第二、三类边值条件: $\frac{\partial \xi}{\partial n}+d \xi=g_{2}, d$ 是非负常数，其形式逼近是

$$
\xi_{n}(Q)+\frac{d}{2}\left(\xi(Q)+\xi\left(Q^{\prime}\right)\right)=g_{2}\left(\frac{Q+Q^{\prime}}{2}\right), \quad Q \in R_{h} .
$$

定理 4 假设格式(5)，(7)中的 $v_{0}>0, d^{*}=\operatorname{sign}(d)$, 条件 $(\sigma, \lambda, 0)$ 成立. 那末, 当 $\left\|\tilde{f}_{2}\right\|^{2} \leqslant N h^{2},\left\|\tilde{g}_{2}\right\|^{2} \leqslant N h^{3-d^{*}}, \rho^{*}=\rho\left(\tilde{\eta}_{1}, \tilde{f}_{1}, \tilde{f}_{2}, h^{-\frac{1}{2}+\frac{d^{*}}{2}} \tilde{g}_{2}, K\right) \leqslant N h^{2}$ 时, 对一切 $K \tau \leqslant T \leqslant$ $T_{0}\left(\rho^{*}\right)$ 者, $\|\tilde{\eta}(K)\|_{0 \oplus v}^{2} \leqslant M e^{L T} \rho^{*}$. 
若把 $f_{i}, \tilde{x}_{2}$ 看作形式逼近误差, 则当 $\left\|f_{1}\right\|^{2}=o(1),\left\|f_{2}\right\|^{2}=O\left(h^{2}\right),\left\|\tilde{g}_{2}\right\|^{2}=O\left(h^{3-d^{*}}\right)$ 时， 格式收敛. 在实际预报中, 还遇到下列外插边界条件

$$
\eta\left(Q^{\prime}\right)=\eta(Q), \quad Q \in R_{h}^{*},
$$

或

$$
\eta\left(Q^{\prime}\right)=2 \eta(Q)-\eta\left(Q^{\prime \prime}\right), \quad Q \in R_{h}^{*},
$$

其中 $Q^{\prime \prime}$ 表示 $R_{h}$ 内, 法向上距 $Q$ 为 $h$ 的点. (8),(9)式在形式上可看作条件(7)式,其中 $d=0$, 且分别有形式逼近误差 $\tilde{g}_{2}=O(1)$ 或 $\tilde{g}_{2}=O(h)$. 因此, 定理 4 无法保证其收玫性. Platzman 和 Mastnuo 的计算结果也证实至少应采用三层以上内点值来外插,计算才稳定.

有时还采用定常边值条件,但往往尽管计算稳定,但预报值向平稳态过渡. 事实上，假设 (4)的解 $\xi, \psi$ 与下列定常流的解 $\xi^{*}, \psi^{*}$ 具有相同的定常边值条件, 例如零边值条件, 又设 $v=$ 常数 $>0$,

$$
\left\{\begin{array}{l}
\frac{\partial}{\partial x_{1}}\left(\nu \frac{\partial \xi^{*}}{\partial x_{1}}\right)+\frac{\partial}{\partial x_{2}}\left(\nu \frac{\partial \xi^{*}}{\partial x_{2}}\right)+\frac{\partial \psi^{*} \partial \xi^{*}}{\partial x_{2} \partial x_{1}}-\frac{\partial \psi^{*}}{\partial x_{1}} \frac{\partial \xi^{*}}{\partial x_{2}}=0, \\
\nabla^{2} \psi^{*}-\xi^{*}+f=0 .
\end{array}\right.
$$

作者已证明,在很弱的条件下,(10)式存在广义解. 又暂记 $\|\xi\|^{2}=\iint_{R} \xi^{2} d x_{1} d x_{2},\|\xi\|_{1}^{2}=\left|\frac{\partial \xi}{\partial x_{1}}\right|^{2}$ $+\left\|\frac{\partial \xi}{\partial x_{2}}\right\|^{2}, M^{(2)}$ 题一切有限支集, 无限次光滑函数在范数 $\|\xi\|+\|\xi\|_{1}$ 意义下的闭包, $m_{0}^{*}=$ $\sup _{\xi \in M^{(2)}}\|\xi\|^{2} /\|\zeta\|_{1}^{2}$, 则有

提理 5 若广义雷诺尔数 $R_{e}^{*}=\frac{2 \sqrt{2}}{v^{2}} m_{0}^{* 3 / 2}\|f\|^{2}<1$ 。那末(10)式的广义解唯一, 且 当 $t \rightarrow \infty$ 时, $\left\|\xi(t)-\xi^{*}\right\|^{2} \leqslant e^{-a t}\left\|\xi(0)-\xi^{*}\right\|^{2} \rightarrow 0, \alpha=\nu\left(1-R_{e}^{*}\right) m_{0}^{*-1}$.

因此，采用定常边值条件会导致误报. 然而定理 5 却又是定常流问题动态松驰法的理论 基础，详见另文.

\section{五、可 顶 拫 胡}

可以把差分格式视为对大气过程的直接离散逼近, $f_{a}, f_{0}, f_{c}$ 分别是通近误差,初始条件观 测误差和计算舍入误差, $\tilde{\eta}$ 表示预报误差. 若格式的广义稳定性指标 $S<\infty$, 则当 $\|\tilde{f}\|=\cdot\left\|f_{a}\right\|$ $+\left\|f_{0}\right\|+\left\|f_{c}\right\| \leqslant N h^{s}$ 时, 存在 $T_{0}>0$, 当 $t \leqslant T_{0}\left(\| f f^{\prime}\right)$ 时, $\|\tilde{\eta}(t)\| \leqslant M\|f\|$, 即预报误差 是可控制的，且当 $h \rightarrow 0, \tau \rightarrow 0$ 时, 预报误差趋于零. 或者说至少存在可预报期 $T_{0}$. 由本 文结果得到:

（1）仅当 $\lambda, \delta, \sigma$ 满足一定条件时,才有 $T_{0}>0$. 隐式格式和二步法可增大 $T_{0}$;

（2）参数 $\alpha_{1}=\alpha_{2}$ 和适当的平滑过程可增大 $T_{0}$;

（3）合适的边界形式与边值条件可增大 $T_{0}$,边值误差会减少 $T_{0}$;

（4）由于 $f_{c}$ 与计算机字长有关,因此 $T_{0}$ 与计算工具有关;

（5）由于 $N, f_{a}$ 等与 $\eta, \varphi$ 的某些差商值有关,所以大气变化越激烈, $T_{0}$ 就越小.

本文是在王宗皓先生的建议和帮助下整理的, 并得到冯康和林群老师的支持和帮助, 特此致谢. 